Pacific Journal of Mathematics

ULTRAFILTER AND MAPPING 


\title{
ULTRAFILTERS AND MAPPINGS
}

\section{TAKESI ISIWATA}

\begin{abstract}
We give characterizations of closed, quasi-perfect, $d-, Z-, W Z$, $W^{*}$-open, $N$-, $W N-, W_{r} N$ - and other maps using closed or open ultrafilters and investigate relations between these maps and various properties as generalizations of realcompactness, i.e., almost-, $a$-, $c$ - and $w a$-real compactness, $c b^{*}$-ness and weak $c b^{*}$-ness. Finally we establish several theorems about the perfect $W^{*}$-open image of a weak $c b^{*}$ space and its application to the absolute $E(X)$ of a given space $X$.
\end{abstract}

We characterize closed, $Z$-, $W Z$-, $N$ - and $W N$-maps by closed ultrafilters in $\S 1$ and show that $\varphi$ is $W^{*}$-open iff $\varphi^{\#} U$ is an open ultrafilter for each open ultrafilter $\mathcal{Q}$ in $\$ 2$. In $\$ 3$, introducing the notion of ${ }^{*}$-open map, we show that $\beta \varphi$ is open iff $\varphi$ is a ${ }^{*}$-open $W_{r} N$-map iff there is $\mathcal{Q}^{p}$ with $\varphi^{\# Q^{p}}=\mathcal{V}^{q}$ for each $q \in \beta Y$, each $\mathfrak{V}^{q}$ and each $p \in(\beta \varphi)^{-1} q$. In $\S 4$, we discuss invariance concerning CIP of closed or open ultrafilters under various maps and establish invariances and inverse invariance of various properties as a generalization of realcompactness under suitable maps in $\S 5$. In $\S 6$, we give several theorems about the perfect $W^{*}$-open image of weak $c b^{*}$ spaces which contain, as corollaries, known results concerning the absolute $E(X)$ of $X$.

Throughout this paper, by a space we mean a completely regular Hausdorff space and assume familiarity with [3] whose notion and terminology will be used throughout. We denote by $\varphi: X \rightarrow Y$ a continuous onto map and by $\beta X(v X)$ the Stone-Čech compactification (realcompactification) of $X$ and by $\beta \varphi$ the Stone extension over $\beta X$ of $\varphi$. In the sequel, we use the following notation and abbreviation. $N=$ the set of positive integers, $\mathrm{CIP}=$ countable intersection property, $\mathrm{nbd}=$ neighborhood, $\mathscr{F}^{p}=$ a closed ultrafilter converging to $p$. We denote by $\mathscr{F}(\mathscr{Q})$ a closed (open) ultrafilter on $X$ and by $\mathcal{E}(\mathfrak{V})$ a closed (open) ultrafilter on $Y \cdot \varphi^{\#} \mathscr{F}=\left\{E \subset Y ; \varphi^{-1} E \in \mathscr{F}\right.$ and $E$ is closed in $\left.Y\right\}$. Similarly define $\varphi^{\#}$ Q.

\section{Closed ultrafilters.}

1.1. In the sequel, we use frequently the following results.

(1) If $p \in \cap_{\mathrm{cl}_{\beta X}} \varphi^{-1} \mathcal{F}^{q}=\cap\left\{\mathrm{cl}_{\beta X} \varphi^{-1} E ; E \in \mathcal{E}^{q}\right\}$, then there is $\mathscr{F}^{p}$ with $\varphi^{\# \mathscr{F}^{p}}=\mathcal{E}^{q}$. For, $\mathbb{Q}=\left\{\varphi^{-1} E \cap F ; E \in \mathcal{E}^{q}, F \in N(p)\right\}$ is a closed filter base where $N(p)$ is a closed nbd base of $p$ in $\beta X$. Obviously $Q \rightarrow p$. Thus any $\mathscr{F}^{p}$ containing $\mathscr{Q}$ has the property $\varphi^{\# \mathscr{F}^{p}}=\mathcal{E}^{q}$. It is easily seen that the same method above can be applied to open ultrafilter and 
$Z$-ultrafilter respectively i.e., if $p \in \cap \mathrm{cl}_{\beta X} \varphi^{-1} \mathcal{V}^{q}\left(\cap \mathrm{cl}_{\beta X} \varphi^{-1 \mathscr{Z}^{q}}\right)$, there is $\mathcal{Q}^{p}\left(\mathscr{L}^{p}\right)$ with $\varphi^{\#} \mathcal{Q}^{p}=\mathcal{V}^{q}\left(\varphi^{\# \mathscr{L}^{p}}=\mathscr{Z}^{q}\right)$.

(2) For $x \in X$, a closed ultrafilter $\mathscr{F}$ converging to $x$ is unique and $\mathscr{F}=\{F ; x \in F$ and $F$ is closed $\}$. Obviously $\{x\} \in \mathscr{F}$. It is easy to see that $X$ is normal iff for each $p \in \beta X$, a closed ultrafilter $\mathscr{F}$ converging to $p$ is unique and $\mathscr{F}=\left\{F ; p \in \operatorname{cl}_{\beta X} F\right.$ and $F$ is closed $\}$.

(3) For $p \in \beta X$, a $Z$-ultrafilter $\mathscr{Z}^{p}$ is unique and $\mathscr{Z}^{p}=\{Z ; Z$ is a zero set and $p \in \mathrm{cl}_{\beta X} Z$ \}.

1.2. Let $\varphi: X \rightarrow Y,(\beta \varphi) p=q, p \in \beta X$ and $q \in \beta Y$.

(1) $\cap \mathrm{cl}_{\beta Y} \varphi^{\# \mathscr{F}^{p}}=\{q\}$.

(2) $\varphi^{-1} \mathcal{E}^{q} \subset \mathscr{F}^{p} \Leftrightarrow \varphi^{\# \mathscr{F}^{p}}=\mathcal{E}^{q}$.

(3) $\cap \mathrm{cl}_{\beta X} \varphi^{-1} \mathscr{G}^{q} \subset(\beta \varphi)^{-1} q$.

(4) $\cap \mathrm{cl}_{\beta X} \varphi^{-1} \mathcal{E}^{y}=\mathrm{cl}_{\beta X} \varphi^{-1} y$ for $y \in Y$.

(5) $\varphi^{\# \mathscr{F}^{p}} \subset \mathcal{E}^{q} \Leftrightarrow \operatorname{cl}(\varphi F) \cap E \neq \varnothing$ for $F \in \mathscr{F}^{p}$ and $E \in \mathcal{E}^{q}$.

(6) There is $\mathscr{F}^{p}$ such that $\varphi^{\# \mathscr{F}^{p}}$ is a closed ultrafilter iff there is $\mathcal{E}^{q}$ with $p \in \cap \mathrm{cl}_{\beta X} \varphi^{-1} \mathcal{E}^{q}$.

Proof. (1) It suffices to show that $\cap \mathrm{cl}_{\beta Y} \varphi^{\# \mathscr{F}^{p}}$ consists of only one point. Let $q_{i} \in \cap \mathrm{cl}_{\beta Y} \varphi^{\# \mathscr{F}^{p}}(i=1,2)$. Then there are disjoint closed nbd's $V_{1}$ and $V_{2}$ of $q_{1}$ and $q_{2}$ in $\beta Y$ respectively, so $X \cap(\beta \varphi)^{-1} V_{i} \in \mathscr{F}^{p}$ $(i=1,2)$, a contradiction.

(2) Obvious.

(3) If $r \in \cap \mathrm{cl}_{\beta X} \varphi^{-1} \mathcal{G}^{q}-(\beta \varphi)^{-1} q$, there is $\mathscr{F}^{r}$ with $\varphi^{-1} \mathcal{F}^{q} \subset \mathscr{F}^{r}$ by 1.1(1) and (2) above. This shows $(\beta \varphi)^{-1} q \ni r$, a contradiction.

(4) From $\{y\} \in \mathcal{E}^{y}$.

$(5) \Rightarrow)$. From $\operatorname{cl}(\varphi F) \in \varphi^{\#} F^{p}$ for $F \in \mathscr{F}^{p}$. $\left.\Leftarrow\right)$. Let $K \in \varphi^{\# \mathscr{F}^{p}}-\mathcal{E}^{q}$. Then $\mathscr{F}=\varphi^{-1} K \in \mathscr{F}^{p}$. Since $K \notin \mathcal{E}^{q}$, there is $E \in \mathcal{E}^{q}$ with $K \cap E=\varnothing$, i.e., $\operatorname{cl}(\varphi F) \cap E=\varnothing$, a contradiciton.

$(6) \Rightarrow)$. Let $\mathscr{E}^{q}=\varphi^{\# \mathscr{F}^{p}}$. Then $\varphi^{-1} \mathscr{G}^{q} \subset \mathscr{F}^{p}$, so $\left.p \in \cap \mathrm{cl}_{\beta X} \varphi^{-1} \mathscr{G}^{q} . \Leftarrow\right)$. From 1.1(1).

1.3. Definition. We recall that $\varphi: X \rightarrow Y$ is a $Z$-map if $\varphi Z$ is closed for every zero set $Z$ and $\varphi$ is a WZ-map if $(\beta \varphi)^{-1} y=\operatorname{cl}_{\beta X} \varphi^{-1} y$ for each $y \in Y$. It is known that a closed map is a $Z$-map and a $Z$-map is $W Z$ [12]. Woods [21] introduced the notions of $N$ - and $W N$-map. $\varphi$ is an $N(W N)$ map if $(\beta \varphi)^{-1} \mathrm{cl}_{\beta Y} R=\mathrm{cl}_{\beta X} \varphi^{-1} R$ for every closed set (zero set) $R$ of $Y$. An $N$-map is $W N$ and $W Z$. In the following, we characterize maps mentioned above by closed ultrafilters.

THEOREM 1.4. Let $\varphi: X \rightarrow Y$.

(1) $\varphi$ is WZ iff there is $\mathscr{F}^{p}$ with $\varphi^{\# \mathscr{F}^{p}}=\mathcal{E}^{y}$ for each $y \in Y$ and each $p \in(\beta \varphi)^{-1} y$.

(2) $\varphi$ is a Z-map iff there is $\mathscr{F}^{p}$ such that $Z \in \mathscr{F}^{p}$ and $\varphi^{\#}=\mathcal{E}^{y}$ for each $y \in Y$, each $p \in(\beta \varphi)^{-1} y$ and each zero set $Z$ with $p \in \mathrm{cl}_{\beta X} Z$. 
(3) The following are equivalent:

(i) $\varphi$ is closed.

(ii) $\varphi^{\#} \mathscr{F}$ is a closed ultrafilter for any $\mathscr{F}$.

(iii) There is $\mathscr{F}^{p}$ such that $F \in \mathscr{F}^{p}$ and $\varphi^{\# \mathscr{F}^{p}}=\mathcal{E}^{y}$ for each $y \in Y$, each $p \in(\beta \varphi)^{-1}$ and each closed set $F$ with $p \in \operatorname{cl}_{\beta X} F$.

(4) The following are equivalent:

(i) $\varphi$ is an $N$-map.

(ii) $(\beta \varphi)^{-1} q=\cap \mathrm{cl}_{\beta X} \varphi^{-1} \mathcal{E}^{q}$ for each $q \in \beta Y$ and each $\mathcal{E}^{q}$.

(iii) There is $\mathscr{F}^{p}$ with $\varphi^{\# \mathscr{F}^{p}}=\mathcal{E}^{q}$ for each $q \in \beta Y$, each $\mathcal{E}^{q}$ and each $p \in(\beta \varphi)^{-1} q$.

(5) The following are equivalent:

(i) $\varphi$ is a WN-map.

(ii) $\mathrm{cl}_{\beta X} \varphi^{-1} \mathscr{Z}^{q}=(\beta \varphi)^{-1} q$ for each $q \in \beta Y$.

(iii) $\varphi^{\# \mathscr{Z}^{p}}=\mathscr{Z}^{q}$ for each $q \in \beta Y$ and each $p \in(\beta \varphi)^{-1} q$.

Proof. $(1) \Rightarrow)$. Since $\varphi$ is $W Z$, we have $(\beta \varphi)^{-1} y=\mathrm{cl}_{\beta X} \varphi^{-1} y$ and $(\beta \varphi)^{-1} y=\cap_{\mathrm{cl}_{\beta X}} \varphi^{-1} \mathcal{E}^{y}$ by 1.2(4). Thus there is $\mathscr{F}^{p}$ with $\varphi^{\# \mathscr{F}^{p}}=\mathcal{E}^{y}$ by $1.1(1) \Leftarrow)$. For each $p \in(\beta \varphi)^{-1} y$, we have $p \in \cap \mathrm{cl}_{\beta X} \varphi^{-1} \mathcal{E}^{y}$ by $1.2(6)$. Since $\cap \mathrm{cl}_{\beta X} \varphi^{-1} \mathcal{E}^{y}=\mathrm{cl}_{\beta X} \varphi^{-1} y$ by $1.2(4),(\beta \varphi)^{-1} y \subset \mathrm{cl}_{\beta X} \varphi^{-1} y$, so $(\beta \varphi)^{-1} y$ $=\mathrm{cl}_{\beta X} \varphi^{-1} y$ which shows that $\varphi$ is $W Z$.

$(2) \Rightarrow)$. Let $p \in(\beta \varphi)^{-1} y$ and $Z$ a zero set with $p \in \operatorname{cl}_{\beta X} Z$. Since $\varphi$ is a $Z$-map, $\varphi Z$ is closed, so $y \in \varphi Z$. On the other hand, $\varphi^{-1} y=X \cap$ $\left(\cap \mathrm{cl}_{\beta X} \varphi^{-1} \mathscr{G}^{y}\right)$ by 1.2(4). If $p \in X$, then there is $\mathscr{F}^{p}$ with $\varphi^{\# \mathscr{F}^{p}}=\mathcal{E}^{y}$ by $1.2(6)$ and since $p \in X, p \in Z$ so $Z \in \mathscr{F}^{p}$. Now suppose $p \notin X$. Since $y \in E$ for $E \in \mathcal{E}^{y}$ and $\varphi Z \exists y$, we have $Z \cap \varphi^{-1} E \neq \varnothing$. We shall show $p \in \cap \mathrm{cl}_{\beta X}\left(Z \cap \varphi^{-1} E\right)$ for $E \in \mathcal{E}^{y}$. Suppose contrary. There is a zero set $K$ of $\beta X$ such that $p \in$ int $_{\beta X} K$ and $K \cap \mathrm{cl}_{\beta X}\left(Z \cap \varphi^{-1} E\right)=\varnothing . Z^{\prime}=K \cap$ $Z \neq \varnothing$ and $p \in \mathrm{cl}_{\beta X} Z^{\prime}$, but $y \notin \varphi Z^{\prime}$, a contradiction. Thus there is $\mathscr{F}^{p} \supset\left\{Z \cap \varphi^{-1} E ; E \in \mathcal{E}^{y}\right\}$ by $1.1(1)$. Obviously $\varphi^{-1} \mathscr{G}^{y} \subset \mathscr{F}^{p}$, so $\varphi^{\# \mathscr{F}^{p}}=$ $\mathcal{E}^{y}$ and $Z \in \mathscr{F}^{p}$. $\Leftarrow$ ). Let $Z$ be a zero set and $y \in \operatorname{cl} \varphi Z-\varphi Z$. Then we have $p \in \operatorname{cl}_{\beta X} Z \cap(\beta \varphi)^{-1} y$, so there is $\mathscr{F}^{p}$ with $Z \in \mathscr{F}^{p}$ and $\varphi^{\# \mathscr{F}^{p}}=\mathcal{E}^{y}$. Since $\{y\} \in \mathcal{E}^{y}, \varphi^{-1} y \in \mathscr{F}^{p}$, but $Z \cap \varphi^{-1} y=\varnothing$, a contradiction.

(3) (i) $\Rightarrow$ (ii) $\Rightarrow$ (iii). Evident. (iii) $\Rightarrow$ (i). Suppose that there is a closed set $F$ of $X$ with $y \in \operatorname{cl}(\varphi F)-\varphi F$. Then $K=\operatorname{cl}_{\beta X} F \cap(\beta \varphi)^{-1} y \neq \varnothing$. Let $p \in K$. By (iii), there is $F \in \mathscr{F}^{p}$ with $\varphi^{\# \mathscr{F}^{p}}=\mathcal{E}^{y}$. Since $\{y\} \in \mathcal{E}^{y}$ and $F \in \mathscr{F}^{p}$, we have $F \cap \varphi^{-1} y \neq \varnothing$ which is a contradiction.

(4) (i) $\Rightarrow$ (ii). Since $\varphi$ is an $N$-map and $q \in \mathrm{cl}_{\beta Y} E$ for each $E \in \mathcal{E}^{q}$, we have $(\beta \varphi)^{-1} q \subset \cap(\beta \varphi)^{-1} \mathrm{cl}_{\beta Y} \mathcal{E}^{q}=\cap_{\mathrm{cl}_{\beta X}} \varphi^{-1} \mathcal{E}^{q}$, and hence $(\beta \varphi)^{-1} q=$ $\cap \mathrm{cl}_{\beta X} \varphi^{-1} \mathcal{E}^{q}$ by 1.2(3). (ii) $\Rightarrow$ (iii). From (ii) and 1.2(6). (iii) $\Rightarrow$ (i). Suppose that there is a closed set $E$ of $Y$ with $K=(\beta \varphi)^{-1} \mathrm{cl}_{\beta Y} E-\mathrm{cl}_{\beta X} \varphi^{-1} E \neq \varnothing$. Let $p \in K$ and $(\beta \varphi) p=q$. Then $q \in \operatorname{cl}_{\beta Y} E$. Let $E \in \mathcal{E}^{q}$. Take $\mathscr{F}^{p}$ with $\varphi^{\# \mathscr{F}^{p}}=\mathcal{E}^{q}$. Since $p \notin \mathrm{cl}_{\beta X} \varphi^{-1} E$, we have $\varphi^{-1} E \notin \mathscr{F}^{p}$, a contradiction.

(5) This is proven by the analogous method used in (4) above. 


\section{Open ultrafilters.}

2.1. Let $g: X \rightarrow Y$ and $(\beta \varphi) p=q, p \in \beta X, q \in \beta Y$.

(1) $\cap \mathrm{cl}_{\beta Y} \varphi^{\#} \mathcal{Q}^{p}=\cap \mathrm{cl}_{\beta Y} \varphi^{\mathcal{Q}^{p}}=\{q\}$.

(2) $\varphi^{-1} \mathscr{V}^{q} \subset \mathcal{Q}^{p} \Leftrightarrow \varphi^{\#} \mathcal{Q}^{p}=\mathscr{V}^{q}$.

(3) $\cap \mathrm{cl}_{\beta X} \varphi^{-1} \mathscr{V}^{q} \subset \cap \mathrm{cl}_{\beta X} \varphi^{-1}\left(\mathrm{cl} \mathscr{V}^{q}\right) \subset(\beta \varphi)^{-1} q$.

(4) $\varphi^{\#} \mathcal{Q}^{p} \subset \mathcal{V}^{q} \Leftrightarrow \varphi U \cap \mathrm{cl} V \neq \varnothing$ for $U \in \mathcal{Q}^{p}$ and $V \in \mathcal{V}^{q}$.

(5) There is $\mathcal{Q}^{p}$ such that $\varphi^{\#} \mathcal{Q}^{p}$ is an open ultrafilter iff there is $\mathcal{V}^{q}$ with $p \in \cap \mathrm{cl}_{\beta X} \varphi^{-1 \mathfrak{V} q}$.

The proof of 2.1 is obtained by the same method used in 1.2. By 1.1(1), "if part" of 2.1(5) implies that there is $\vartheta^{p}$ with $\varphi^{\#} Q^{p}=\mathscr{V}^{q}$. As is shown by 2.2 below, it is not necessarily true that if there is $\mathcal{V}^{q}$ with $p \in \cap \mathrm{cl}_{\beta X} \varphi^{-1}\left(\mathrm{cl} \mathcal{V}^{q}\right)$, then there is $\mathscr{Q}^{p}$ with $\varphi^{\#} \mathcal{Q}^{p}=\mathcal{V}^{q}$.

ExAmple 2.2. Let $X=[0,1) \oplus[1,2]$ and $Y=[0,2]$. Define $\varphi: X \rightarrow Y$ by $\varphi(x)=x$ for $x \in X$. Let $\mathcal{V}^{q} \ni[0,1), q=1 \in Y$. Then $p=1 \in$ $\cap \operatorname{cl}_{\beta X} \varphi^{-1}\left(\operatorname{cl}^{q}\right)$ and any $\mathcal{Q}^{p}$ contain $(1,2]$ and hence $\varphi^{\#} \mathcal{Q}^{p} \neq \mathfrak{V}^{q}$ (cf. 3.1 below).

Lemma 2.3. Let $\varphi^{\#} \mathcal{Q}^{p} \subset \mathfrak{V}^{q}, U \in \mathcal{Q}=\mathcal{Q}^{p}, V \in \mathfrak{V}^{q}=\mathfrak{V}$ and let us put $B(U, V)=U \cap \varphi^{-1}(\mathrm{cl} V)$. Then we have

(1) Int $B(U, V) \in$ Q .

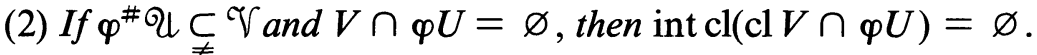

(3) If $\varphi^{\#} \mathscr{Q}=\mathcal{T}$, then int $\operatorname{cl}(\varphi U) \in \mathcal{V}$.

Proof. (1). By 2.1(4), $B(U, V) \neq \varnothing$. Suppose $S=$ int $B(U, V)=\varnothing$. $U-B(U, V)$ is open in $U$, so in $X$. Since $(X-\operatorname{cl} U) \cup(U-B(U, V))$ is dense in $X$ and $\mathcal{Q}$ is prime, we have $U-B(U, V) \in \mathcal{Q}$. But $\varphi^{-1} \operatorname{cl} V \cap$ $(U-B(U, V))=\varnothing$, and hence $\operatorname{cl} V \cap \varphi(U-B(U, V))=\varnothing$, a contradiction by 2.1(4). Thus $S \neq \varnothing$. If $S \notin \mathcal{Q}$, there is $W \in \mathcal{Q}$ with $W \cap$ $S=\varnothing$. This implies $S \cap W=\operatorname{int}\left(U \cap \varphi^{-1}(\operatorname{cl} V) \cap W\right)=$ $\operatorname{int}\left(U \cap W \cap \varphi^{-1}(\operatorname{cl} V)\right)=$ int $B(U \cap W, V)=\varnothing$, a contradiction.

(2) Since $V \cap \varphi U=\varnothing$ implies $V \cap \operatorname{cl}(\varphi U)=\varnothing$, we have

$$
\operatorname{cl}(\varphi U \cap \operatorname{cl} V) \subset \operatorname{cl} \varphi U \cap \operatorname{cl} V \subset \operatorname{cl}(\varphi U) \cap(\operatorname{cl} V-V),
$$

so int $\operatorname{cl}(\varphi U \cap \operatorname{cl} V)=\varnothing$.

(3) If int $\operatorname{cl} \varphi U \notin \mathcal{V}$, we have $Y-\operatorname{cl} \varphi U \in \mathcal{V}$, so $X-\varphi^{-1} \operatorname{cl}(\varphi U) \in$ थ, a contradiction.

THEOREM 2.4. $\varphi^{\#} \bigcup^{p}$ is an open ultrafilter iff int $\operatorname{cl}(\varphi U) \neq \varnothing$ for $U \in \mathcal{Q}^{p}$. 
Proof. $\Rightarrow$ ) Let $\varphi^{\#} Q^{p}=\mathscr{V}^{q}$. Then this follows from 2.3(3). $\left.\leftarrow\right)$. Sup-

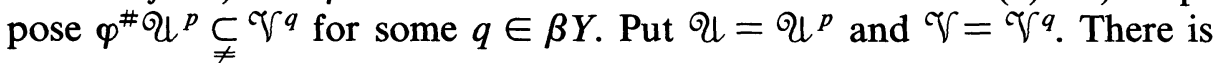
$V \in \mathcal{V}-\varphi^{\#} \mathscr{Q}$ with $V \cap \varphi U=\varnothing$ for some $U \in \mathcal{Q}$. By $2.3(1), W=$ int $B(U, V) \in \mathcal{Q}$ and $\varphi W \cap V=\varnothing$, so int $\operatorname{cl}(\varphi W)=\varnothing$ by 2.3(2), a contradiction.

2.5. Definition. $\varphi: X \rightarrow Y$ is said to be a $W^{*}$-open map if $\operatorname{cl} \varphi U$ is regular closed (i.e., $\operatorname{cl}(\operatorname{int}(\operatorname{cl} \varphi U))=\operatorname{cl} \varphi U)$ whenever $U$ is open [8]. This is a generalization of an open map. We use this notion in the following.

THEOREM 2.6. Let $\varphi: X \rightarrow Y$. The following are equivalent:

(1) $\varphi$ is $W^{*}$-open.

(1') $\mathrm{Cl} \varphi U$ is regular closed whenever $U$ is a basic open set of $X$.

(2) $\operatorname{Int}(\mathrm{cl} \varphi U) \neq \varnothing$ for each non-empty open set $U$ of $X$.

$\left(2^{\prime}\right) \operatorname{Int}(\operatorname{cl} \varphi U) \neq \varnothing$ for each non-empty basic open set $U$ of $X$.

(3) $\operatorname{Int}\left(\mathrm{cl} \varphi^{-1} V\right)=\operatorname{int} \varphi^{-1}(\mathrm{cl} V)$ for each open set $V$ of $Y$.

(4) $\varphi^{\#} Q$ is an open ultrafilter for any $थ$.

(5) There is $\mathcal{Q}^{p}$ such that $\varphi^{\# Q^{p}}$ is an open ultrafilter for each $q \in \beta Y$ and each $p \in(\beta \varphi)^{-1} q$.

(6) $(\beta \varphi)^{-1} q=\cup\left\{\cap_{c X} \varphi^{-1} \mathfrak{V} ; \mathcal{V}\right.$ is an open ultrafilter converging to q) for each $q \in \beta Y$.

Proof. (1) $\Rightarrow\left(1^{\prime}\right) \Rightarrow\left(2^{\prime}\right) \Leftrightarrow(2)$ and (4) $\Rightarrow(5)$ are evident. (2) $\Leftrightarrow(4)$. From $2.4(5) \Leftrightarrow(6)$. From 2.1(3, 5).

(2) $\Rightarrow$ (3). It suffices to show int $\varphi^{-1} \operatorname{cl} V \subset \operatorname{cl}\left(\varphi^{-1} V\right)$. Suppose $x \in$ int $\varphi^{-1}(\operatorname{cl} V)-\operatorname{cl}\left(\varphi^{-1} V\right)$. There is an open set $W \ni x$ such that $W \cap$ $\operatorname{cl}\left(\varphi^{-1} V\right)=\varnothing$ and $W \subset$ int $\varphi^{-1}(\operatorname{cl} V)$. Then $V \cap \varphi W=\varnothing$, so $V \cap \operatorname{cl} \varphi W$ $=\varnothing$. On the other hand, $\varphi W \subset \operatorname{cl} V$, so int $(\operatorname{cl} \varphi W) \subset \operatorname{cl} V-V$ and hence int $\operatorname{cl}(\varphi W)=\varnothing$, a contradiction.

(5) $\Rightarrow(2)$. Let $U \subset X$ be open and $x \in U$. Then any open ultrafilter $थ$ converging to $x$ contains $U$. There is $\mathcal{U}^{x}$ such that $\varphi^{\#} U^{x}$ is an open ultrafilter by (5). Thus int $\operatorname{cl} \varphi U \neq \varnothing$ by 2.4 .

(3) $\Rightarrow(2)$. Suppose that there is an open set $U$ with int $\operatorname{cl} \varphi U=\varnothing$. Let us put $V=Y-\operatorname{cl} \varphi U$. Then $\operatorname{cl} V=Y$ and int $\varphi^{-1}(\operatorname{cl} V)=X$. But $\operatorname{int}\left(\operatorname{cl} \varphi^{-1} V\right) \cap U=\varnothing$, a contradiction.

$(2) \Rightarrow(1)$. Let $U$ be open and put $K=\operatorname{clint}(\operatorname{cl} \varphi U)$. Suppose $y \in \varphi U$ $-K$. Then there is an open set $W \ni y$ with $K \cap \operatorname{cl} W=\varnothing$. Since $T=U \cap \varphi^{-1} W \neq \varnothing$ and $\operatorname{cl} \varphi T \subset \operatorname{cl} W \cap \operatorname{cl} \varphi U$, int $\operatorname{cl}(\varphi T) \subset \operatorname{int}(\operatorname{cl} W)$ $\cap \operatorname{int}(\operatorname{cl} \varphi U)=\varnothing$, a contradiction. Thus $\varphi U \subset K$ and hence $\operatorname{cl} \varphi U \subset K$, i.e., $\operatorname{cl} \varphi U=K$. 


\section{3. *-open mappings.}

3.1. Definition. $\varphi: X \rightarrow Y$ is said to be *-open if $\operatorname{int}(\operatorname{cl} \varphi U) \supset \varphi U$ for each open set $U$ of $X$. An open map is *-open but a *-open map is not necessarily open by 3.2 below. A *-open map is $W^{*}$-open by 2.6 but a $W^{*}$-open map is not necessarily *-open by 2.2 in which it is easy to see that $\varphi$ is $W^{*}$-open. Let $U=[1,2] \subset X$. Then $U$ is open in $X$ and $\operatorname{int}(\operatorname{cl} \varphi U)=(1,2] \not \supset \varphi U=[1,2]$, so $\varphi$ is not *-open (cf. 5.6 below). We say that $\varphi$ is a $W_{r} N$-map if $\operatorname{cl}_{\beta X} \varphi^{-1} R=(\beta \varphi)^{-1} \mathrm{cl}_{\beta Y} R$ for every regular closed set $R$ of $Y$ [10]. $X$ is almost normal [17] ( $\kappa$-normal [16]) if each regular closed set is completely separated from each closed (regular closed) set disjoint from it.

EXAMPLE 3.2. Let $P$ be the set of rational numbers in $[0,1], X=$ $[0,1] \oplus P, Y=[0,1]$ and $\varphi(x)=x \in Y$ for each $x \in X$. Then $\varphi$ is not open. To show that $\varphi$ is *-open, it suffices to prove that $\operatorname{int}(\operatorname{cl} \varphi U) \supset \varphi U$ for each open set $U$ of $P$. Let $U \subset P$ be open. There is an open set $W \subset[0,1]$ with $P \cap W=U . W$ is the union of countably many disjoint open interval $W_{n}=\left(a_{n}, b_{n}\right)$. Put $P_{n}=P \cap W_{n}$. Obviously $\operatorname{cl} \varphi P_{n}=$ $\left[a_{n}, b_{n}\right]$ and $\operatorname{int}\left(\operatorname{cl} \varphi P_{n}\right) \supset P_{n}$, so $\operatorname{int}(\operatorname{cl} \varphi U) \supset \varphi U$, i.e., $\varphi$ is *-open.

THEOREM 3.3. Let $\varphi: X \rightarrow Y$. The following are equivalent:

(1) $\varphi$ is *-open.

(2) $\mathrm{Cl} \varphi^{-1} V=\varphi^{-1} \mathrm{cl} V$ for each open set $V$ of $Y$.

(3) $\cap \mathrm{cl}_{\beta X} \varphi^{-1} \mathscr{V}^{y} \supset \mathrm{cl}_{\beta X} \varphi^{-1} y$ for each $y \in Y$ and each $\mathcal{V}^{y}$.

(4) There is $\mathcal{Q}^{p}$ with $\varphi^{\#} \mathcal{Q}^{p}=\mathcal{V}^{y}$ for each $y \in Y$, each $p \in \operatorname{cl}_{\beta X} \varphi^{-1} y$ and each $\mathcal{V}^{y}$.

Proof. $(1) \Rightarrow(2)$. Suppose that there is an open set $V$ of $Y$ with $x \in \varphi^{-1} \operatorname{cl} V-\operatorname{cl} \varphi^{-1} V$. Take an open set $W \ni x$ disjoint from $\operatorname{cl} \varphi^{-1} V$. Since $V \cap \operatorname{cl} \varphi W=\varnothing$ and $\varphi$ is *-open, we have $\operatorname{int}(\operatorname{cl} \varphi W) \cap \operatorname{cl} V=\varnothing$ and $\operatorname{int}(\operatorname{cl} \varphi W) \supset \varphi W \ni \varphi(x)$, a contradiction.

(2) $\Rightarrow(3)$. Take $\Upsilon^{y}$. Since $\operatorname{cl}_{\beta X} \varphi^{-1} V=\operatorname{cl}_{\beta X} \varphi^{-1}(\operatorname{cl} V)$ and $y \in \operatorname{cl} V$ for $V \in \mathcal{V}^{y}$, we have $\varphi^{-1} y \subset \cap \mathrm{cl}_{\beta X} \varphi^{-1} \mathcal{V}^{y}$, so $\mathrm{cl}_{\beta X} \varphi^{-1} y \subset \cap \mathrm{cl}_{\beta X} \varphi^{-1} \mathcal{V}^{y}$.

(3) $\Rightarrow$ (4). From 2.1(5).

$(4) \Rightarrow(1)$. Suppose that there is an open set $U$ with $x \in U$ and $y=\varphi(x) \in \varphi U-\operatorname{int}(\operatorname{cl} \varphi U)$. Let $W \ni y$ be open. Then $V=W \cap(Y-$ $\operatorname{cl} \varphi U) \neq \varnothing, y \notin V$ and $y \in \operatorname{cl} V$. Take $\mathcal{T}^{y} \ni V$. Any $\mathcal{Q}^{x}$ contains $U$. If $\varphi^{\#} \mathcal{Q}^{x}=\mathcal{V}^{y}$ for some $\mathcal{Q}^{x}$, then $\varphi^{-1} V \in \mathcal{Q}^{x}$, but $\varphi^{-1} V \cap U=\varnothing$, a contradiction.

In general the equality in 3.3(3) does not hold by 3.8 below. From the definition of a $W Z$-map, 2.1(3) and 3.3(3) we have 
Corollary 3.4. If $\varphi: X \rightarrow Y$ is *-open $W Z$, then $(\beta \varphi)^{-1} y=$ $\cap \mathrm{cl}_{\beta X} \varphi^{-1} \mathfrak{V}^{y}$ for each $y \in Y$ and each $\mathfrak{V}^{y}$.

EXAMPLE 3.5. We give an example which shows that the converse of 3.4 is not necessarily true. Let $X=\left[0, \omega_{1}\right] \oplus\left[0, \omega_{1}\right), Y=\left[0, \omega_{1}\right]$ and $\varphi(x)=x \in Y$ for each $x \in X$ where $\omega_{1}$ is the first uncountable ordinal. Then $\varphi$ is open but not WZ. It is easy to see $(\beta \varphi)^{-1} y=\cap_{\mathrm{cl}_{\beta X}} \varphi^{-1} \mathfrak{V}^{y}$ for each $y \in Y$ and each $\mathfrak{T}^{y}$.

THEOREM 3.6. $\varphi: X \rightarrow Y$ is $W_{r} N$ iff $(\beta \varphi)^{-1} q=\cap_{c_{\beta X}} \varphi^{-1} \mathrm{cl} \mathfrak{V}^{q}$ for each $q \in \beta Y$ and each $\mathfrak{V}$ ?

Proof. $\Rightarrow)$. Since $\mathrm{cl}_{\beta X}\left(\varphi^{-1} \mathrm{cl} V\right)=(\beta \varphi)^{-1} \mathrm{cl}_{\beta Y} V$ for $V \in \mathbb{V}^{q},(\beta \varphi)^{-1} q$ $\subset \cap \mathrm{cl}_{\beta X} \varphi^{-1} \mathrm{cl} \mathfrak{V}^{q}$, so we have the equality by $\left.2.1(3) . \leftarrow\right)$. Let $p \in$ $(\beta \varphi)^{-1} \operatorname{cl}_{\beta Y} V-\operatorname{cl}_{\beta X} \varphi^{-1} \mathrm{cl} V$ for some open set $V$ of $Y$. Then $p \in(\beta \varphi)^{-1} q$ for some $q \in \operatorname{cl}_{\beta Y} V$. Take $\mathfrak{V}^{q}$ with $V \in \mathfrak{V}^{q}$. Then $\operatorname{cl}_{\beta X} \varphi^{-1} \operatorname{cl} V \not \supset(\beta \varphi)^{-1} q$, a contradiction.

THEOREM 3.7. (1) The following are equivalent ([10], Theorems 1 and 6):

(i) $Y$ is almost normal.

(ii) Any WZ-map onto $Y$ is $W_{r} N$.

(iii) Any perfect map onto $Y$ is $W_{r} N$.

(2) The following are equivalent:

(i) $Y$ is k-normal.

(ii) Any $W^{*}$-open WZ-map onto $Y$ is $W_{r} N$.

(iii) Any $W^{*}$-open perfect map onto $Y$ is $W_{r} N$.

Proof. (2) (i) $\Rightarrow$ (ii). Let $\varphi: X \rightarrow Y$ be $W^{*}$-open and $W Z$. Suppose $p \in(\beta \varphi)^{-1} \operatorname{cl}_{\beta Y} V-\operatorname{cl}_{\beta X} \varphi^{-1} \mathrm{cl} V$ for some open set $V$ of $Y$. Then $(\beta \varphi) p$ $=q \in \operatorname{cl}_{\beta Y} V$ and take an open set $W$ of $\beta X$ such that $p \in W$ and $\operatorname{cl}_{\beta X} W \cap \mathrm{cl}_{\beta X} \varphi^{-1} \mathrm{cl} V=\varnothing$. Since $\varphi$ is $W^{*}$-open and $W Z$, we have that $(\beta \varphi) \mathrm{cl}_{\beta X} W \cap \mathrm{cl} V=\varnothing$ and $\mathrm{cl} \varphi(X \cap W)$ is regular closed. Thus cl $\varphi(X \cap W) \cap \operatorname{cl} V=\varnothing$, and hence $\operatorname{cl}_{\beta Y} \varphi(X \cap W) \cap \mathrm{cl}_{\beta Y} V=\varnothing$ because $Y$ is $\kappa$-normal. On the other hand, $\operatorname{cl}_{\beta X}(X \cap W)=\mathrm{cl}_{\beta X} W \ni p$, so $q \in \mathrm{cl}_{\beta X} \varphi(X \cap W) \cap \mathrm{cl}_{\beta Y} V$, a contradiction. (ii) $\Rightarrow$ (iii). Evident.

(iii) $\Rightarrow$ (i). This follows from the same method used in 1.5 of [21]. Suppose that there are disjoint regular closed sets $E$ and $K$ such that $\operatorname{cl}_{\beta Y} E \cap \operatorname{cl}_{\beta Y} K \ni q$. Let $X=Y \oplus E$. Define $\varphi: X \rightarrow Y$ by $\varphi(x)=x$ for $x \in X$. It is evident that $\varphi$ is $W^{*}$-open perfect. On the other hand, $\operatorname{cl}_{\beta X} \varphi^{-1} K=\operatorname{cl}_{\beta Y} K$ and $(\beta \varphi)^{-1} \mathrm{cl}_{\beta Y} K \cap \beta E \neq \varnothing$, so $(\beta \varphi)^{-1} \mathrm{cl}_{\beta Y} K \neq$ $\operatorname{cl}_{\beta X} \varphi^{-1} K$ which shows that $\varphi$ is not $W_{r} N$. 
EXAMPLE 3.8. In 3.7(2,ii), “ $W Z$-ness of $\varphi$ " is necessary as shown by the following. Let $Y=[0,3], X=[0,2) \oplus(1,3]$ and $\varphi(x)=x$ for $x \in X$. Then $\varphi$ is open and $Y$ is metrizable. $\varphi^{-1}(1)=1$ and $(\beta \varphi)^{-1} 1 \neq \operatorname{cl}_{\beta X} \varphi^{-1}(1)$ $=1$ and hence $\varphi$ is not $W Z$. Let $Y \ni y=1$ and $\mathcal{V}^{y} \ni[0,1)$. Then it is obvious $\cap \mathrm{cl}_{\beta X} \varphi^{-1} \mathrm{cl} \mathcal{V}^{y}=\{1\} \subsetneq(\beta \varphi)^{-1} y$. Thus $\varphi$ is not $W_{r} N$ by 3.6 and hence $\beta \varphi$ is not open by 3.10 below. But $\beta \varphi$ is $W^{*}$-open by Theorem 4 of [7]. Let $Y \ni z=2$ and $\mathcal{V}^{z} \ni[0,2)$. Then it is easy to see that $\cap \operatorname{cl}_{\beta X} \varphi^{-1} \mathcal{V}^{z}$ $\supsetneqq \mathrm{cl}_{\beta X} \varphi^{-1} z=\{2\}$ (cf. Remark of 3.3).

THEOREM 3.9. If $\varphi: X \rightarrow Y$ is $a^{*}$-open Z-map, then it is open.

Proof. Let $U$ be open in $X$ and $x \in U$. Then there is a zero set $Z$ with $x \in$ int $Z=W \subset Z \subset U$ and $\varphi U \supset \varphi Z=\operatorname{cl} \varphi Z \supset \operatorname{cl} \varphi($ int $Z) \supset$ $\operatorname{int}(\operatorname{cl} \varphi($ int $Z)) \supset \varphi W \ni \varphi(x)$, and hence $\varphi$ is open.

THEOREM 3.10. Let $\varphi: X \rightarrow Y$. Then the following are equivalent:

(1) $\beta \varphi$ is open.

(2) $\varphi$ is *-open and $W_{r} N$.

(3) $\mathrm{Cl}_{\beta X} \varphi^{-1} V=(\beta \varphi)^{-1} \mathrm{cl}_{\beta Y} V$ for each open set $V$ of $Y$.

(4) $(\beta \varphi)^{-1} q=\cap \mathrm{cl}_{\beta X} \varphi^{-1} \mathcal{V}^{q}$ for each $q \in \beta Y$ and each $\mathcal{V}^{q}$.

(5) There is $\mathcal{Q}^{p}$ with $\varphi^{\#} \mathcal{Q}^{p}=\mathcal{V}^{q}$ for each $q \in \beta Y$, each $\mathcal{V}^{q}$ and each $p \in(\beta \varphi)^{-1} q$.

Proof. (1) $\Rightarrow(2)$. Let $U$ be open in $X$ and put $W=\beta X-\operatorname{cl}_{\beta X}(X-U)$. Then $U=W \cap X$ and $\operatorname{cl}_{\beta X} W=\operatorname{cl}_{\beta X} U$. Since $\beta \varphi$ is closed, we have $(\beta \varphi) \mathrm{cl}_{\beta X} W=\mathrm{cl}_{\beta Y}(\beta \varphi) U=\mathrm{cl}_{\beta Y} \varphi U \supset(\beta \varphi) W \supset \varphi U$ and $\operatorname{cl} \varphi U=Y \cap$ $\mathrm{cl}_{\beta Y} \varphi U \supset Y \cap(\beta \varphi) W \supset \varphi U$. Since $\beta \varphi$ is open, int $(\operatorname{cl} \varphi U) \supset \varphi U$, i.e., $\varphi$ is *-open. We shall show that $\varphi$ is $W_{r} N$. Let $V$ be open in $Y . T=\beta Y-$ $\operatorname{cl}_{\beta Y}(Y-V)$ is open and $V=Y \cap T$. Since $\operatorname{cl}_{\beta Y} T=\operatorname{cl}_{\beta Y} V$ and $\beta \varphi$ is *-open, $\mathrm{cl}_{\beta X}(\beta \varphi)^{-1} T=(\beta \varphi)^{-1} \mathrm{cl}_{\beta Y} T=(\beta \varphi)^{-1} \mathrm{cl}_{\beta Y} V$. Thus it suffices to show $\operatorname{cl}_{\beta X}(\beta \varphi)^{-1} T=\operatorname{cl}_{\beta X} \varphi^{-1} \mathrm{cl} V$. Suppose $p \in(\beta \varphi)^{-1} T-\operatorname{cl}_{\beta X} \varphi^{-1} \mathrm{cl} V$. Let $q \in T$ and $(\beta \varphi) p=q$. Take an open set $S$ of $\beta X$ such that $S \ni p$ and $\operatorname{cl}_{\beta X} S \cap \operatorname{cl}_{\beta X} \varphi^{-1} \mathrm{cl} V=\varnothing$. Let us put $K=\operatorname{int}_{\beta Y}\left((\beta \varphi) \operatorname{cl}_{\beta X} S\right)$. Then $K=$ $\operatorname{int}_{\beta Y}\left(\operatorname{cl}_{\beta Y}(\beta \varphi) S\right) \supset(\beta \varphi) S \ni q$ and $K \cap V=\varnothing$, so $K \cap \mathrm{cl}_{\beta Y} V=\varnothing$. This is a contradiction because $q \in \mathrm{cl}_{\beta Y} V$. (2) $\Rightarrow(3)$. From 3.3(2). (3) $\Rightarrow$ (4). From 2.1(3) and the fact that $q \in \operatorname{cl}_{\beta Y} V$ for each $V \in \mathcal{V}^{q}$. (4) $\Rightarrow$ (5). From 2.1(5).

$(5) \Rightarrow(1)$. We first show that $\beta \varphi$ is *-open. Let $p \in(\beta \varphi)^{-1} \mathrm{cl}_{\beta Y} W-$ $\operatorname{cl}_{\beta X}(\beta \varphi)^{-1} W$ for some open set $W$ of $\beta Y$. Then there is an open set $U$ of $\beta X$ with $p \in \operatorname{int}_{\beta X} \mathrm{cl}_{\beta X} U$ and $\operatorname{cl}_{\beta X} U \cap \mathrm{cl}_{\beta X}(\beta \varphi)^{-1} W=\varnothing$. Let $(\beta \varphi) p=$ $q$ and take $\mathcal{V}^{q}$ with $W \in \mathcal{V}^{q}$. Then any $\mathcal{Q}^{p}$ contains $U$. If $\varphi^{\#} \mathcal{U}^{p}=\mathfrak{V}^{q}$ for some $\mathcal{Q}^{p}$, then $\varphi^{-1} W \in \mathcal{U}^{p}$, but $U \cap \varphi^{-1} V=\varnothing$, a contradiction. Thus $\beta \varphi$ is *-open by 3.3 , so open by 3.9 . 
If $\varphi: X \rightarrow Y$ is open $W Z$, then $\beta \varphi$ is open by Theorem 4.4(1) of [12]. Let $X \subset Z \subset \beta X$ and $\zeta=(\beta \varphi) \mid Z$. Then $\zeta: Z \rightarrow \zeta Z$ has the Stone extension $\beta \zeta=\beta \varphi$, so $\beta \zeta$ is open, and hence $\zeta$ is *-open $W_{r} N$ by 3.10. Thus we have

THEOREM 3.11. Let $\varphi: X \rightarrow Y$ be open $W Z$. Then for any space $Z, X \subset Z \subset \beta X, \zeta: Z \rightarrow \zeta Z \subset \beta Y$ is ${ }^{*}$-open $W_{r} N$ where $\zeta=(\beta \varphi) \mid Z$.

\section{Countable intersection property.}

4.1. Definition. We denote by $\left\{F_{n}\right\}_{\mathrm{cl}} \downarrow \varnothing\left(\left\{F_{n}\right\}_{z e} \downarrow \varnothing\right.$ or $\left\{F_{n}\right\}_{r e} \downarrow \varnothing$ resp.) a decreasing sequence of closed sets (zero sets or regular closed sets resp.) with empty intersection. $\varphi: X \rightarrow Y$ is said to be a $d\left(d^{\prime}\right.$ or $d^{*}$ resp.)-map if $\cap \mathrm{cl} \varphi F_{n}=\varnothing$ for each $\left\{F_{n}\right\}_{\mathrm{cl}} \downarrow \varnothing\left(\left\{F_{n}\right\}_{r e} \downarrow \varnothing\right.$ or $\left\{F_{n}\right\}_{z e} \downarrow \varnothing$ resp.) $[5,8,11]$. Obviously a $d$-map is $d^{\prime}$ and a $d^{\prime}$-map is $d^{*}([8]$, Theorem 7). We say that $\varphi$ is hyper-real if $(\beta \varphi)(\beta X-v X) \subset \beta Y-v Y$. A hyper-real map is $d^{*}[11]$ (cf. the diagram of 5.4 below). Let us put $X^{*}=\beta X-X$.

$F(X ; 0)=\left\{p \in X^{*} ;\right.$ any $\mathscr{F}^{p}$ has CIP $\}$.

$F(X ; 0, \Delta)=\left\{p \in X^{*} ;\right.$ there is $\mathscr{F}_{1}^{p}$ with CIP and $\mathscr{F}_{2}^{p}$ without CIP $\}$.

$F(X, \Delta)=\left\{p \in X^{*}\right.$; any $\mathscr{F}^{p}$ does not have CIP $\}$.

$F(X ; v, \Delta)=(v X-X) \cap F(X ; \Delta)$.

Similarly we define $U(X ; 0), U(X ; 0, \Delta), U(X ; \Delta)$ and $U(X ; v, \Delta)$ using free open ultrafilters. It is known that $\beta X-v X \subset U(X ; \Delta), U(X ; \Delta) \subset$ $F(X ; \Delta)$ and $F(X ; 0) \subset U(X ; 0)[13]$. Concerning invariance of CIP under a map, we note the following. Let $\varphi: X \rightarrow Y$.

(1) If $\mathscr{Q}$ has CIP, then any $\mathfrak{V} \supset \varphi^{\#} \mathscr{Q}$ has CIP by $2.3(1)$ where " $Q$ has CIP" means " $\cap \operatorname{cl} U_{n} \neq \varnothing$ for $U_{n} \in \mathcal{Q}$ ". Thus, in general, for $\varphi: X \rightarrow Y$, we have $U(Y ; \Delta) \cap(\beta \varphi)(U(X ; 0) \cup U(X ; 0, \Delta))=\varnothing$ and hence $(\beta \varphi)^{-1} U(Y ; \Delta) \subset U(X ; \Delta)$.

(2) If $\mathscr{F}$ has CIP and $\varphi^{\#} \mathscr{F}=\mathcal{E}$, then $\mathcal{E}$ has CIP. This follows from $\varphi^{-1} E \in \mathcal{F}$ for $E \in \mathcal{E}$.

(3) The following (a) and (b) are not necessarily true as is shown by 4.2 below.

(a) $\varphi^{\#} \mathscr{Q}=\mathscr{V}$ does not have CIP for $\mathcal{Q}$ without CIP.

(b) $\varphi^{\#} \mathscr{F}=\mathcal{E}$ does not have CIP for $\mathscr{F}$ without CIP.

Problem. Does $\mathcal{E} \supset \varphi^{\#} \mathscr{F}$ have CIP whenever $\mathscr{F}$ has CIP?

4.2. ExAmple. Let $Y=\{y\}$. In (1) and (2) below, define $\varphi(x)=y$. Then $\varphi$ is open, closed, $R C$-preserving, $Z$-preserving and an $N$-map where $\varphi$ is $R C(Z)$-preserving if $\varphi E$ is regular closed (a zero) set whenever $E$ is a regular closed set (a zero set). 
(1) Let $X$ be pseudocompact but not countably compact. Then $\varphi$ is a $d^{\prime}$-map but not a $d$-map. Evidently there is $\mathscr{F}$ without CIP but $\varphi^{\#} \mathscr{F}=\{y\}$ has CIP.

(2) Let $X$ be a non-pseudocompact space. Then $\varphi$ is not a $d^{*}$-map. Evidently there is $Q$ without CIP but $\varphi^{\#} \mathscr{Q}=\{y\}$ has CIP. It is easy to construct an $N$-map which is not a $d^{*}$-map by taking a suitable space $X$.

THEOREM 4.3. Let $\varphi: X \rightarrow Y$. The following are equivalent:

(1) $\varphi$ is a d-map.

(2) If $\mathcal{F}$ does not have CIP, so neither does any $\mathcal{E} \supset \varphi^{\#} \mathscr{F}$.

(3) $(\beta \varphi)^{-1}(Y \cup F(Y ; 0)) \subset X \cup F(X, 0)$.

(4) $(\beta \varphi)^{-1} Y \subset X \cup F(X ; 0)$.

Proof (1) $\Rightarrow(2)$. From the fact that $\cap \mathrm{cl} \varphi F_{n}=\varnothing$ for $\left\{F_{n} \in \mathscr{F}\right\} \downarrow \varnothing$ and $\operatorname{cl} \varphi F_{n} \in \mathcal{E}$.

$(2) \Rightarrow(3)$. There is $\mathscr{F}^{p}$ without CIP for $p \in F(X ; \Delta) \cup F(X ; 0, \Delta)$, so every $\mathcal{E} \supset \varphi^{\# \mathscr{F}^{p}}$ does not have CIP by $(2)$ and hence $(\beta \varphi) p \notin Y \cup$ $F(Y, 0)$, so $(\beta \varphi)^{-1}(Y \cup F(Y ; 0)) \subset X \cup F(X ; 0)$.

(3) $\Rightarrow$ (4). Evident.

(4) $\Rightarrow(1)$. Let $\left\{F_{n}\right\}_{\mathrm{cl}} \downarrow \varnothing$ and $y \in \cap \mathrm{cl} \varphi F_{n}$. Then $\mathrm{cl}_{\beta X} F_{n} \cap(\beta \varphi)^{-1} y \neq$ $\varnothing$ for $n \in N$. Take $p \in\left(\cap \operatorname{cl}_{\beta X} F_{n}\right) \cap(\beta \varphi)^{-1} y$ and $\mathscr{F}^{p}$ with $F_{n} \in \mathscr{F}^{p}$, $n \in N$. Then $p \in F(X ; 0)$ by (4) but $\mathscr{F}^{p}$ does not have CIP, a contradiction.

REMARK. In general, the equality of 4.3(3) does not hold as shown by 5.6 below. An analogous theorem concerning a $d^{*}$ - and $d^{\prime}$-map was obtained respectively (see, $4.4(2,3)$ below). A closed $d$-map is precisely quasi-perfect (= closed and each fiber is countably compact), so we have the following 4.4(1) using 1.4(3) and 4.3.

4.4. Let $\varphi: X \rightarrow Y$. (1) $\varphi$ is quasi-perfect iff $\varphi^{\#} \mathscr{F}$ is a closed ultrafilter for each $\mathcal{F}$ and $\varphi^{\#} \mathcal{F}$ does not have CIP for each $\mathcal{F}$ without CIP.

(2) $\varphi$ is a $d^{*}$-map iff $(\beta \varphi)^{-1} Y \subset \mathcal{Q} X[11]$.

(3) $\varphi$ is a d'-map iff $(\beta \varphi)^{-1} Y \subset X \cup U(X ; 0)$ [5].

4.5. Let $\varphi: X \rightarrow Y$.

(1) Let $\varphi$ be a $d^{\prime}$-map and $\varphi^{\#} \mathscr{Q}=\mathfrak{V}$. If $\mathcal{Q}$ does not have CIP, then neither does $\mathcal{V}$.

(2) If $\varphi$ is not a $d^{\prime}$-map, there is $\mathcal{Q}$ without CIP such that every Vつ $\varphi^{\#}$ थ has CIP.

(3) If $\varphi$ is $W^{*}$-open, then $\varphi$ is a $d^{\prime}$-map iff $\varphi^{\#}$ Q does not have CIP for each थ without CIP (cf., 4.6(2)). 
Proof. (1) Since $\mathcal{Q}$ does not have CIP, there is $\left\{U_{n} \in \mathcal{Q}\right\} \downarrow$ with $\cap \operatorname{cl} U_{n}=\varnothing$. If $\mathscr{V}$ has CIP, $Y-\operatorname{cl} \varphi U_{n} \in \mathcal{V}$ for some $n . \varphi^{\#} Q=\mathscr{V}$ implies $\varphi^{-1}\left(Y-\operatorname{cl} \varphi U_{n}\right)=X-\varphi^{-1}\left(\operatorname{cl} \varphi U_{n}\right) \in \mathcal{Q}$, a contradiction.

(2) Since $\varphi$ is not $d^{\prime}$, there is $\left\{U_{n}\right\}_{\text {open }} \downarrow \varnothing$ with $y \in \cap \mathrm{cl} \varphi U_{n}$ for some $y \in Y$. This implies $(\beta \varphi)^{-1} y \cap \mathrm{cl}_{\beta X} U_{n} \neq \varnothing$ for $n \in N$. By 1.1(2), there is $\mathcal{Q}^{p}$ without CIP and $U_{n} \in \mathcal{Q}^{p}$ where $p \in\left(\cap \operatorname{cl}_{\beta X} U_{n}\right) \cap(\beta \varphi)^{-1} y$. Obviously any $\mathcal{V} \supset \varphi^{\#} Q^{p}$ converges to $y$, i.e., $\mathcal{V}$ has CIP.

$(3) \Rightarrow$ ). From (1) and $2.6 \Leftarrow)$. From (2) and 2.6.

4.6. Definitions and some properties. Let $\varphi: X \rightarrow Y . \varphi$ is said to be an $s d$-map if $\mathscr{F}$ does not have CIP iff no $\mathscr{E} \supset \varphi^{\#} \mathscr{F}$ has CIP. We say that $\varphi$ is an $s d^{\prime}$-map if some $\mathfrak{V} \supset \varphi^{\#} Q$ does not have CIP for $Q$ without CIP.

(1) A quasi-perfect map is $s d$ by 4.4 and an $s d$-map is $d$ by 4.3 . $4.5(2)$.

(2) Any $W^{*}$-open $d^{\prime}$-map is $s d^{\prime}$ by $4.5(3)$ and an $s d^{\prime}$-map is $d^{\prime}$ by

(3) If $\varphi$ is $s d$, then we have that $(\beta \varphi)^{-1}(Y \cup F(Y ; 0)) \subset X \cup F(X ; 0)$, $(\beta \varphi) F(X ; 0, \Delta) \subset F(Y ; 0, \Delta)$ and $(\beta \varphi) F(X ; \Delta) \subset F(Y ; \Delta) \cup F(Y ; 0, \Delta)$.

(4) If $\varphi$ is $s d^{\prime}$, then we have that $(\beta \varphi)^{-1}(Y \cup U(Y ; 0)) \subset X \cup$ $U(X ; 0),(\beta \varphi) U(X ; 0, \Delta) \subset U(Y ; 0, \Delta)$ and $(\beta \varphi) U(X ; \Delta) \subset U(Y ; \Delta) \cup$ $U(Y ; 0, \Delta)$.

(5) If $\varphi$ is *-open $W_{r} N$, then $(\beta \varphi)^{-1} U(Y ; 0, \Delta) \subset(X ; 0, \Delta)$, $(\beta \varphi)^{-1} U(Y ; \Delta) \subset U(X, \Delta)$ and $(\beta \varphi) U(X ; 0) \subset Y \cup U(Y ; 0)$ by 3.10 and 4.1(1).

(6) If $\varphi$ is a ${ }^{*}$-open $W_{r} N d^{\prime}$-map, then $(\beta \varphi)^{-1} U(Y ; \Delta)=U(X ; \Delta)$ by 3.10. $(\beta \varphi)^{-1} U(Y ; 0, \Delta)=U(X ; 0, \Delta)$ and $(\beta \varphi)^{-1}(Y \cup U(Y ; 0)=X \cup$ $U(X ; 0)$.

(7) If $\varphi$ is closed, then $(\beta \varphi)(F(X ; 0) \cup F(X ; 0, \Delta)) \cap F(Y ; \Delta)=\varnothing$ by $1.4(3)$ and $4.1(2)$.

(8) If $\varphi$ is an $N$-map, then we have $(\beta \varphi) F(X ; 0) \cap(F(Y ; 0, \Delta) \cup$ $F(Y ; \Delta))=\varnothing$ by $1.1(1)$ and $1.4(4)$.

It is not necessarily true that a perfect map is $s d^{\prime}$ as shown by 4.7 below. $X$ is said to be $n d-c p$ if for a decreasing sequence $\left\{F_{n}\right\}$ of nowhere dense closed sets with $\cap F_{n}=\varnothing$, there is $\left\{U_{n}\right\}_{\text {open }} \downarrow$ with $F_{n} \subset U_{n}$ and $\cap \mathrm{cl} U_{n}=\varnothing$. It is easy to see the following

(9) If $X$ is countably paracompact, then $X$ is $n d-c p$.

(10) If $X$ is pseudocompact, then $X$ is countably compact iff $X$ is $n d-c p$.

4.7. If $Y$ is pseudocompact but not countably compact, then there is a space $X$ and a perfect map $\varphi: X \rightarrow Y$ which is neither sd' nor $W^{*}$-open. 
Proof. Let $A=\left\{a_{n} ; n \in N\right\}$ be a discrete closed set of $Y$ and put $X=Y \oplus A$. Define $\varphi(x)=x$. Obviously $\varphi$ is perfect but not $W^{*}$-open. Let us put $U_{n}=\left\{a_{m} ; m \geq n\right\} \subset A \subset X$ and take $\mathcal{Q}$ with $U_{n} \in \mathcal{Q}, n \in N$. Then $\mathcal{U}$ does not have CIP but any $\mathscr{V} \supset \varphi^{\#} \mathcal{Q}$ has CIP because $Y$ is pesudocompact.

THEOREM 4.8. Let $\varphi: X \rightarrow Y$.

(1) If $Y$ is countably compact, then $X$ is countably compact iff $\varphi$ is sd.

(2) If $Y$ is pseudocompact, then $X$ is pseudocompact iff $\varphi$ is $s d^{\prime}$.

4.8(2) is a generalization of 4.3 of [12] and Theorem 12 of [8].

Proof. $(1) \Rightarrow$ ). Evident. $\Leftarrow)$. If $X$ is not countably compact, there is $\left\{F_{n}\right\}_{\mathrm{cl}} \downarrow \varnothing$. Take $\mathscr{F} \ni F_{n}$ for each $n$. Then $\mathscr{F}$ does not have CIP and hence there is $\mathcal{E}$ without CIP containing $\varphi^{\# \mathscr{F}}$ because $\varphi$ is $s d$. But this is a contradiction because $Y$ is countably compact.

(2) is obtained by the same method used in the proof of (1).

THEOREM 4.9. Let $\varphi: X \rightarrow Y$ and $Y$ be $n d-c p$.

(1) If $\varphi$ is $d^{\prime}$, then $\varphi$ is $s d^{\prime}$.

(2) If $\varphi$ is $d$, then $\varphi$ is $s d$.

Proof. (1). Suppose that there is $Q$ without CIP such that each $\mathcal{V} \supset \varphi^{\#} \mathscr{Q}$ has CIP. If $\varphi^{\#} \mathcal{Q}=\mathfrak{V}$, then $\mathcal{V}$ does not have CIP by 4.5(1), and hence we may assume that $\varphi^{\#} \mathcal{Q} \neq \mathfrak{V}$ for each $\mathfrak{V} \supset \varphi^{\#} \mathcal{Q}$. Since $\mathcal{Q}$ does not have CIP, there is $\left\{U_{n} \in Q\right\} \downarrow \varnothing$ with $\cap \operatorname{cl} U_{n}=\varnothing . \varphi$ being $d^{\prime}$, $\cap \operatorname{cl} \varphi U_{n}=\varnothing$. Let $V \in \mathscr{V}-\varphi^{\#} \mathcal{Q}$. Then there is $U \in \mathcal{Q}$ with $U \cap \varphi^{-1} V$ $=\varnothing$ and hence we may assume $U_{n} \subset U$ for each $n$. Now $\varphi B\left(U_{n}, V\right) \subset$ $\varphi U_{n} \cap \mathrm{cl} V$, so by 2.3(2) $K_{n}=\operatorname{cl} \varphi\left(\right.$ int $\left.B\left(U_{n}, V\right)\right)$ is nowhere dense and $\cap K_{n}=\varnothing$. Since $Y$ is $n d-c p$, there is $\left\{V_{n}\right\}_{\text {open }} \downarrow \varnothing$ such that $K_{n} \subset V_{n}$ and $\cap \mathrm{cl} V_{n}=\varnothing$. Obviously $\varphi^{-1} V_{n} \supset$ int $B\left(U_{n}, V\right)$, so $V_{n} \in \mathcal{V}$ by $2.3(1)$ which shows that $\mathcal{V}$ does not CIP, a contradiction.

(2) By 4.3, it suffices to show that if $\mathscr{F}$ has CIP, then any $\mathscr{E} \supset \varphi^{\#} \mathscr{F}$ has CIP. Suppose that $\mathscr{F}$ has CIP and some $\mathscr{E} \supset \varphi^{\#} \mathscr{F}$ does not have CIP. We may assume $\mathcal{E} \neq \varphi^{\#} \mathscr{F}$. There is $\left\{E_{n} \in \mathcal{E}-\varphi^{\#} \mathscr{F}\right\} \downarrow \varnothing$. Then there is $F \in \mathcal{F}$ with $E_{1} \cap \varphi F=\varnothing$, and hence $E_{n} \cap \varphi F=\varnothing$ for each $n$. Since $\mathcal{E} \ni K_{n}=E_{n} \cap \operatorname{cl} \varphi F \neq \varnothing$ and $K_{n}$ is nowhere dense, there is $\left\{V_{n}\right\}_{\text {open }} \downarrow \varnothing$ such that $K_{n} \subset V_{n}$ and $\cap \operatorname{cl} V_{n}=\varnothing$. If $\operatorname{cl} V_{n} \notin \varphi^{\# \mathscr{F}}$, then there is $D \in \mathscr{F}$ with $\operatorname{cl} V_{n} \cap \varphi D=\varnothing$. $V_{n}$ being open, $V_{n} \cap \operatorname{cl} \varphi D=\varnothing$ and hence $K_{n} \cap$ $\operatorname{cl} \varphi D=\varnothing$ which contradicts $\mathcal{E} \supset \varphi^{\# \mathscr{F}}$. This shows $\operatorname{cl} V_{n} \subset \varphi^{\#} \mathscr{F}$ for each $n$, so $F$ does not have CIP, a contradiction. 


\section{Spaces and mappings.}

5.1. We recall the following [13].

(1) $X$ is almost realcompact iff $U(X ; 0) \cup U(X ; 0, \Delta)=\varnothing$.

(2) $X$ is $c$-realcompact iff $U(X ; 0)=\varnothing$.

(3) $X$ is $a$-realcompact iff $F(X ; 0) \cup F(X ; 0, \Delta)=\varnothing$.

(4) $X$ is wa-realcompact iff $F(X ; 0)=\varnothing$.

(5) $X$ is weak $c b^{*}$ iff $U(X ; v, \Delta) \cup U(X ; 0, \Delta)=\varnothing$.

(6) $X$ is pseudocompact iff $U(X ; \Delta) \cup U(X ; 0, \Delta)=\varnothing$.

(7) $X$ is $c b^{*}$ iff $F(X ; v, \Delta) \cup F(X ; 0, \Delta)=\varnothing$.

(8) $X$ is countably compact iff $F(X ; \Delta) \cup F(X ; 0, \Delta)=\varnothing$.

Dykes and Frolik proved the following respectively.

(9) Let $\varphi: X \rightarrow Y$ be perfect. Then

(i) $X$ is almost realcompact iff $Y$ is almost realcompact [2].

(ii) $X$ is $a$-realcompact iff $Y$ is $a$-realcompact [1].

From $(1) \sim(8)$, we have the following diagram.

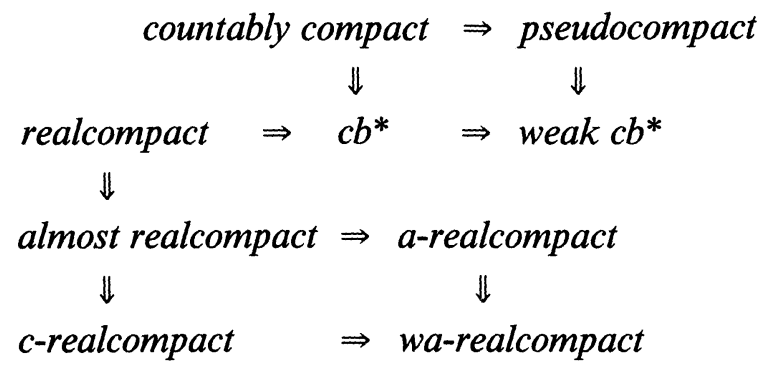

5.2. Let $p \in X^{*}, Z=X \cup\{p\} \subset \beta X$ and $Y$ the space obtained from $Z$ by identifying $p$ and a fixed point $x_{0}$ of $X$. It is easy to see that the identifying map $\varphi$ is $W^{*}$-open but not ${ }^{*}$-open. In this case we have

(1) If $p \in \mathscr{V} X-X$, then $\varphi$ is $d^{*}[\mathbf{1 1}]$.

(2) If $p \in U(X ; 0)$, then $\varphi$ is $d^{\prime}[5]$.

THEOREM 5.3. (1) The following are equivalent:

(i) $X$ is wa-realcompact.

(ii) Any d-map defined on $X$ is perfect.

(iii) Any $W^{*}$-open sd-map defined on $X$ is perfect.

(2) The following are equivalent ([5], Theorem 1 and [8], Theorem 13):

(i) $X$ is c-realcompact.

(ii) Any $d^{\prime}$-map defined on $X$ is perfect.

(iii) Any $W^{*}$-open $d^{\prime}$-map defined on $X$ is perfect.

(3) The following are equivalent ([11], Theorem 6.3):

(i) $Y$ is $c b^{*}$.

(ii) Any $d^{*}$-map onto $Y$ is hyper-real.

(iii) Any perfect map onto $Y$ is hyper-real. 
(4) The following are equivalent:

(i) $Y$ is weak $c b^{*}$.

(ii) Any sd'-map onto $Y$ is hyper-real.

(iii) Any $W^{*}$-open $d^{\prime}$-map onto $Y$ is hyper-real.

(iv) Any $W^{*}$-open perfect map onto $Y$ is hyper-real.

Proof. (1) (i) $\Rightarrow$ (ii). From 4.3(2,3) and wa-realcompactness. (ii) $\Rightarrow$ (iii). Evident. (iii) $\Rightarrow$ (i). If $X$ is not wa-realcompact, take $p \in F(X ; 0)$ in 5.2. Obviously $\varphi$ is $W^{*}$-open $s d$-map but $\varphi^{-1}\left(x_{0}\right)=x_{0}$ and $(\beta X)^{-1} x_{0} \ni p$, so $\varphi$ is not perfect.

(4) (i) $\Rightarrow$ (ii). Since $\varphi$ is $s d^{\prime},(\beta \varphi)(\beta X-v X) \subset(\beta \varphi) U(X ; \Delta) \cup$ $U(Y ; \Delta) \cup U(Y ; 0, \Delta)=\beta Y-v Y$ because $Y$ is weak $c b^{*}$, i.e., $\varphi$ is hyperreal. (ii) $\Rightarrow$ (iii). From $4.6(2)$. (iii) $\Rightarrow$ (iv). Evident. (iv) $\Rightarrow$ (i). Suppose that there is $\mathcal{Q}^{p}$ without CIP and $p \in v Y-Y$. There is $\left\{U_{n} \in \mathcal{Q}^{p}\right\} \downarrow \varnothing$ with $\cap \operatorname{cl} U_{n}=\varnothing$. Let us put $X=Y \oplus \Sigma \oplus \operatorname{cl} U_{n}$ and define $\varphi(x)=x$. Obviously $\varphi$ is $W^{*}$-open perfect. On the other hand, $v X=v Y \oplus \Sigma \oplus v\left(\operatorname{cl} U_{n}\right)$ and $v \varphi$ is onto $v Y$, but $(v \varphi)^{-1} p(p \in v Y)$ is not compact where $v \varphi=$ $(\beta \varphi) \mid(v X)$, and hence $\varphi$ is not hyper-real.

5.4. Note AND Problem. We define that $\varphi: X \rightarrow Y$ is a $d_{1}\left(d_{2}\right)$-map if $(\beta \varphi)^{-1} Y \subset X \cup U(X ; 0) \cup U(X ; 0, \Delta)(\subset X \cup F(X ; 0) \cup F(X ; 0, \Delta))$. Then we have the following:

(1) $X$ is almost realcompact iff any $d_{1}$-map defined on $X$ is perfect.

(2) $X$ is a-realcompact iff any $d_{2}$-map defined on $X$ is perfect. "only if" part of (1) and (2) are obvious and "if" part of (1) and (2) are obtained by the method used in 5.2 taking $p \in U(X ; 0, \Delta) \cup U(X ; 0)$ and $p \in F(X ; 0, \Delta) \cup F(X ; 0)$ respectively. But these definitions of $d_{1^{-}}$and $d_{2}$-map are affected.

Problem. What is the intrinsic definition of a $d_{1}$ (or $d_{2}$ )-map? Concerning various maps in this paper, we have the following:

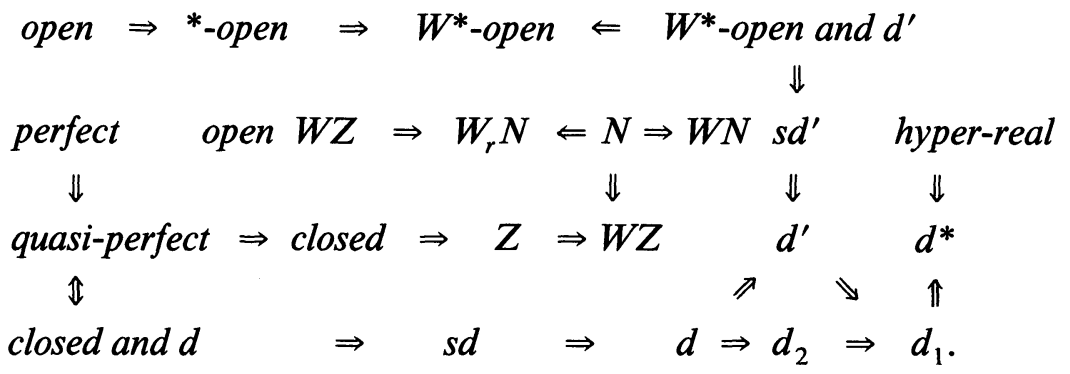

THEOREM 5.5. Let $\varphi: X \rightarrow Y$.

(1) Suppose that $\varphi$ is a d-map. Then we have

(i) If $X$ is wa-realcompact, so is $Y$.

(ii) If $X$ is a-realcompact, so is $Y$. 
(2) Let $\varphi$ be an sd'-map. Then if $X$ is c-realcompact, so is $Y$ (this is a generalization of Theorem 1.3 of [7] by $4.6(2)$ ).

(3) Let $\varphi$ be a d'-map. Then if $X$ is almost realcompact, so is $Y$.

(4) Let $\varphi$ be hyper-real. Then if $X$ is weak $c b^{*}$, so is $Y$.

(5) Let $\varphi$ be hyper-real. Then if $X$ is $c b^{*}$, so is $Y([11]$, Theorem 5.7(2)).

Proof. (1) (i). From 5.1(4), 5.3(1) and 4.3(3) (note that a perfect map is $s d)$. (ii). From the diagram of 5.1, 5.3, (i) above and 5.1(9(ii)).

(2) From $U(Y ; 0)=\varnothing$ by $4.6(4)$ and $U(X ; 0)=\varnothing$, or from 4.6(4), Theorem 2 of [4] and the fact that $u X=X \cup U(X ; 0)$.

(3) From the diagram of 5.1, 5.3(2) and 5.1(9(i)).

(4) Suppose that there is $\mathcal{V}^{q}$ without CIP for $q \in v Y-Y$. Then $(\beta \varphi)^{-1} q \subset U(X ; 0)$. Take $p \in(\beta \varphi)^{-1} q$ and $\mathcal{Q}^{p} \supset \varphi^{-1} \mathcal{V}^{q}$. Since $\mathcal{Q}^{p}$ has CIP, so does $\varphi^{\#} Q^{p}=\mathcal{V}^{q}$, a contradiction. Thus $U(Y ; v, \Delta) \cup$ $U(Y ; 0, \Delta)=\varnothing$, so $Y$ is weak $c b^{*}$.

Since a compact space is realcompact, by $4.2(1,2)$, it is easily seen that almost-, $c$-, $a$ - and wa-realcompactness, $c b^{*}$-ness and weak $c b^{*}$-ness are not inverse invariant under an open, closed, $Z$-preserving, $N$-map. Moreover, by the following Example 5.6, we have that (1) $c$-realcompactness is not inverse invariant under a $W^{*}$-open perfect map and (2) $c b^{*}$-ness and weak $c b^{*}$-ness are not invariant under a $W^{*}$-open perfect map.

5.6. EXAMPLE. K. Morita [15] constructed an $M$-space, non $c$-realcompact space $X$ and a perfect map $\varphi$ such that the perfect image $Y$ [14] of $X$ by $\varphi$ is not an $M$ space. It is easy to see that $\varphi$ is $W^{*}$-open but not *-open. An $M$-space is $c b^{*}$ and hence weak $c b^{*}$. On the other hand, $Y$ is $c$-realcompact [6] but neither $a$-realcompact [22] nor weak $c b^{*}$ [11] and $v Y-Y=U(Y ; 0, \Delta)=F(Y ; 0, \Delta)$ consists of only one point (see [12, 15]). We note that $(\beta \varphi)^{-1}(Y \cup F(Y ; 0))=(\beta \varphi)^{-1} Y \neq X \cup F(X ; 0)$ (cf. Remark of 4.3 and Remark 6.4 below).

THEOREM 5.7. Let $\varphi: X \rightarrow Y$.

(1) Let $\varphi$ be an $s d^{\prime}-m a p$. Then if $Y$ is weak $c b^{*}$, so is $X$.

(2) Let $\varphi$ be a d-map. Then if $Y$ is $c b^{*}$, so is $X([11]$, Theorem 5.5).

(3) Let $\varphi$ be a d'-map and $Y$ almost realcompact. Then we have

(i) $U(X ; 0, \Delta)=\varnothing$.

(ii) If $X$ is c-realcompact, then $X$ is almost realcompact.

(iii) If $\varphi$ is perfect, then $X$ is almost realcompact (5.1(9)).

(4) Let $\varphi$ be an sd-map and Y a-realcompact. Then we have

(i) $F(X ; 0, \Delta)=\varnothing$.

(ii) If $X$ is wa-realcompact, then $X$ is a-realcompact.

(iii) If $\varphi$ is perfect, then $X$ is a-realcompact (5.1(9)). 
(5) Let $\varphi$ be a perfect open map. If $Y$ is a c-realcompact, so is $X$ ([5], Theorem 4).

(6) Let $\varphi$ be a perfect $N$-map. Then if $Y$ is wa-realcompact, so is $X$.

Proof. (1) $\varphi$ being hyper-real, by 5.3(4) $\beta X-v X=(\beta \varphi)^{-1}(\beta Y-v Y)$ and $U(X ; v, \Delta) \cup U(X ; 0, \Delta)=\varnothing$ by 4.6(4) and 5.1(5), and hence $X$ is weak $c b^{*}$.

(3) (i). By 4.1(1) and 4.4(3), $(\beta \varphi) U(X ; 0, \Delta) \subset U(Y ; 0, \Delta)$ and hence we have $U(X ; 0, \Delta)=\varnothing$ because $Y$ is almost realcompact. (ii). From (i) and 5.1(1,2). (iii). (New proof) Let $p \in U(X ; 0)$. Then any $\mathcal{T} \supset \varphi^{\#} Q^{p}$ has CIP and converges to a point $q \in v Y-Y$ by 4.1(1) and $X=(\beta \varphi)^{-1} Y$. Since $Y$ is almost realcompact, $v Y-Y=U(Y ; v, \Delta)$, a contradiction. Our assertion follows from (i) and 5.1(1).

(4) (i). By 4.6(3), $(\beta \varphi) F(X ; 0, \Delta) \subset F(Y ; 0, \Delta)$, so $F(X ; 0, \Delta)=\varnothing$ and hence $X$ is $a$-realcompact because $Y$ is $a$-realcompact. (ii). From (i) and 5.1(3,4). (iii). (New proof) Let $p \in F(X ; 0)$. Since $\varphi$ is $s d$, some $\mathcal{E} \supset \varphi^{\# \mathscr{F}}$ has CIP and converges to a point $q \in v Y-Y$ by $X=(\beta \varphi)^{-1} Y$. Since $Y$ is $c$-realcompact, $v Y-Y=F(Y ; v, \Delta)$, a contradiction. Our assertion follows from (i) and 5.1(3).

(5) (New proof) From 4.6(6) and $X=(\beta \varphi)^{-1} Y$.

(6) Since $\varphi$ is $N(\beta \varphi) F(X ; 0) \subset Y \cup F(Y ; 0)=Y$ by 4.6(8), and since $\varphi$ is perfect $(\beta \varphi)^{-1} Y=X$ and $F(Y ; 0)=\varnothing$ because $Y$ is $w a$-realcompact and hence $X$ is wa-realcompact.

6. Weak $c b^{*}$-ness and absolute. Using preceding results we give new proofs of several theorems concerning the absolute $E(X)$ of $X$ which are obtained as corollaries of theorems about perfect $W^{*}$-open images of weak $c b^{*}$ spaces.

THEOREM 6.1. Let $\varphi$ be a perfect $W^{*}$-open map of a weak $c b^{*}$ space $X$ onto $Y$. Then we have

(1) $\varphi$ is hyper-real iff $Y$ is weak $c b^{*}$.

(2) $(\beta \varphi) \cup X=Y \cup U(Y ; 0) \cup U(Y ; 0, \Delta)$.

(3) $X$ is realcompact iff $Y$ is almost realcompact.

(4) $v X=(\beta \varphi)^{-1} T$ for some $T$ with $Y \subset T \subset \beta Y$ iff $T=Y \cup U(Y$; 0$)$ and $U(Y ; 0, \Delta)=\varnothing$.

Proof. (1) From 5.3(4) and 5.5(4).

(2) Suppose $(\beta \varphi)^{-1} q \subset \beta X-v X$ for some point $q \in U(Y ; 0) \cup$ $U(Y ; 0, \Delta)$. Then there is $\mathcal{V}^{q}$ with CIP and $\mathcal{U}^{p}$ with $\varphi^{\#} \mathcal{Q}^{p}=\Upsilon^{q}$ for $p \in(\beta \varphi)^{-1} q$. Since $\mathcal{Q}^{p}$ does not have CIP and $\varphi$ is $s d^{\prime}, \mathcal{T}^{q}$ does not have CIP, a contradiction. 
$(3) \Rightarrow)$. Since $\varphi$ is perfect and $X=v X$, we have $U(Y, 0) \cup$ $U(Y ; 0, \Delta)=\varnothing$ by $(2)$, so $Y$ is almost realcompact $\Leftarrow)$. Since $Y$ is almost realcompact $(\beta \varphi) v X=Y$ by (2). On the other hand, $(\beta \varphi)^{-1} Y=X$, and hence $v X=X$, i.e., $X$ is realcompact.

$(4) \Rightarrow)$. By (2), we have $(\beta \varphi) \cup X=T=Y \cup U(Y ; 0) \cup U(Y ; 0, \Delta)$. Since $\varphi$ is perfect and $W^{*}$-open, $\varphi$ is $s d^{\prime}$ and $(\beta \varphi)^{-1}(Y \cup U(Y ; 0)) \subset X \cup$ $U(X ; 0)=v X$ by $4.6(4)$. We shall show $U(Y ; 0, \Delta)=\varnothing$. Let $q \in$ $U(Y ; 0, \Delta)$. Then $(\beta \varphi)^{-1} q \subset U(X ; 0)$ and there is ${ }^{q}{ }^{q}$ without CIP but any $\mathcal{Q}^{p}$ has CIP for each $p \in(\beta \varphi)^{-1} q$. Since $\varphi$ is $W^{*}$-open, $\varphi^{\#} \mathcal{Q}^{p}=\mathcal{V}^{q}$ for some $p \in(\beta \varphi)^{-1} q$ and some $\mathcal{Q}^{p}$ and hence $\mathcal{V}^{q}$ has CIP by 4.1(1), a contradiction $\Leftarrow)$. By $(2),(\beta \varphi) \cup X=Y \cup U(Y ; 0) \cup U(Y ; 0, \Delta)=Y \cup$ $U(Y ; 0)$. Since $\varphi$ is $s d^{\prime},(\beta \varphi) U(X ; \Delta) \subset U(Y ; \Delta) \cup U(Y ; 0, \Delta)=U(Y, \Delta)$ by 4.6(4). Thus $(\beta \varphi)^{-1} T=v X$ where $T=Y \cup U(Y ; 0)$.

Let $E(X)$ be the set of all fixed open ultrafilters on $X$ topologized by using $\left\{U^{0} ; U\right.$ is open in $\left.X\right\}$ as a basis where $U^{0}=\{\mathscr{Q} ; U \in \mathscr{Q}\} . E(X)$ is called the absolute of $X$ and it is a Hausdorff extremally disconnected space. Define $\eta: \eta \mathscr{U}=\cap \mathrm{cl} \mathscr{Q}$. Then it is known that $\eta$ is a perfect irreducible map and $\beta E(X)=E(\beta X)$. Since $\eta U^{0}=\operatorname{cl} U[18], \eta$ is $W^{*}$ open by $2.6(2)$. We note that an extremally disconnected space is weak $c b^{*}$.

Corollary 6.2. (1) $v E(X)=(\beta \eta)^{-1} v X(=E(v X))$ iff $u X=v X([7]$, Theorem 2.4 and $[8]$, Theorem 4.2) iff $X$ is weak $c b^{*}$.

(2) $(\beta \eta) v E(X)=a_{1} X([22]$, Lemma 2.1).

(3) $E(X)$ is realcompact iff $X$ is almost realcompact [1].

(4) $v E(X)=(\beta \eta)^{-1} T$ for some $T$ with $X \subset T \subset \beta X$ iff $T=X \cup$ $U(X ; 0)$ and $U(X ; 0, \Delta)=\varnothing([20], p .330$ and [22], Theorem 3.3).

(5) $E(X)$ is pseudocompact iff $X$ is pseudocompact ([20], Proposition 2.5).

Proof. We note that $E(X)$ is weak $c b^{*}$ and $\eta$ is perfect $W^{*}$-open. (1) Since $u X=\left\{p \in \beta X\right.$; each $\mathcal{Q}^{p}$ has CIP $\}$ ([7], Lemma 2.5) and $u X=X \cup$ $U(X ; 0)$ by 4.4 , we have that $v X=u X$ iff $X$ is weak $c b^{*}$. Thus (1) follows from 6.1(1). (2) From 6.1(2) and $a_{1} X=X \cup U(X ; 0) \cup U(X ; 0, \Delta)$ ([22], Theorem 2.3). (3) From 6.1(3). (4) From 6.1(4). (5) From 4.6(2) and 4.8(2).

THEOREM 6.3. Let $\varphi$ be a perfect $W^{*}$-open map of a non-realcompact $c b^{*}$ space $X$ onto $Y$. Then we have

(1) $Y$ is $c b^{*}$ iff $\varphi$ is hyper-real.

(2) If $Y$ is weak $c b^{*}$ then $Y$ is $c b^{*}$.

(3) If $v Y=Y \cup\{q\}$, then $Y$ is not weak $c b^{*}$ iff $Y$ is c-realcompact but not a-realcompact. 
Proof. (1) From 5.3(3) and 5.5(5). (2) Since $Y$ is weak $c b^{*}, \varphi$ is hyper-real by 5.3(4), so $Y$ is $c b^{*}$ by 5.5(5) because $X$ is $c b^{*}$.

(3) $\Rightarrow)$. By 5.1(5) and $v Y=Y \cup\{q\}$, we have $U(Y ; 0)=\varnothing$, so $Y$ is $c$-realcompact by 5.1(2). On the other hand, $(\beta \varphi) F(X ; 0) \subset F(Y ; 0) \cup$ $F(Y ; 0, \Delta)=F(Y ; 0, \Delta)$ because $F(Y ; 0) \subset U(Y ; 0)=\varnothing$. Thus $Y$ is not $a$-realcompact $\Leftarrow$ ). From realcompactness $=$ (weak $c b^{*}$-ness $)+(c$ realcompactness).

6.4. REMARK. The space $X$ in Example 5.6 is not weak $c b^{*}[11]$ and $Y$ is a perfect $W^{*}$-open image of an $M$-space (we note that an $M$-space is $\left.c b^{*}\right)$. Thus $Y$ is $c$-realcompact but not $a$-realcompact by 6.5(3). On the other hand, this assertion follows also from the following Corollary 6.7 since $\varphi: X \rightarrow Y$ in 5.6 is irreducible [5].

COROLlaRY 6.5. Let $\varphi$ be a perfect irreducible map of a non-realcompact $c b^{*}$ space $X$ onto $Y$ with $v Y=Y \cup\{q\}$. Then $Y$ is not weak $c b^{*}$ iff $Y$ is c-realcompact but not a-realcompact.

Proof. By Proposition 1.9 of [19], $X$ and $Y$ are co-absolute, so $E(X)$ and $E(Y)$ are homeomorphic. Since $X$ is $c b^{*}, E(X)$ is $c b^{*}$ by 5.6(2), so $E(Y)$ is also. Since the canonical map: $E(Y) \rightarrow Y$ is perfect and $W^{*}$-open, we have our assertion by 6.3(3).

THEOREM 6.6. (1) If $V$ is an open set of $Y$ with pseudocompact closure, then any $\mathcal{V}^{q} \ni V$ has CIP.

(2) Let $\varphi: X \rightarrow Y$ be $W^{*}$-open and $d^{\prime}$. Then $S=\beta X-(\beta \varphi)^{-1} v Y$ is dense in $\beta X-v X$ and $\beta Y-(\beta \varphi) \mathrm{cl}_{\beta X} S \subset Y \cup U(Y ; 0)$ (this is a generalization of Theorem 2.8 of [20]).

(3) Let $v Y$ be locally compact. Then we have

(i) $Y$ is weak $c b^{*}[4]$.

(ii) If $\varphi: X \rightarrow Y$ is $s d^{\prime}$, then $\varphi$ is hyper-real.

(iii) $E(v Y)=v E(Y)([20]$, Proposition 2.10).

Proof. (1) Suppose that there is $\left\{V_{n} \in \mathscr{V}^{q}\right\} \downarrow$ with $\cap \mathrm{cl} V_{n}=\varnothing$. Then we have $\left\{\operatorname{cl}\left(V \cap V_{n}\right)\right\} \varnothing$ which contradicts the pseudocompactness of $\operatorname{cl} V$.

(2) Suppose $p \in(\beta X-v X)-\mathrm{cl}_{\beta X} S$. Then any $\mathcal{Q}^{p}$ does not have CIP, so $\varphi^{\# Q^{p}}=\mathcal{V}^{q}$ for some $\mathcal{V}^{q}, q \in v Y-Y$ and hence $\mathcal{V}^{q}$ does not have CIP by 4.5(1). There is $U \in \mathcal{Q}^{p}$ and an open set $W$ of $\beta X$ such that $W \cap X=U$ and $\operatorname{cl}_{\beta X} W \cap \operatorname{cl}_{\beta X} S=\varnothing$. By 2.3(3), int $(\operatorname{cl} \varphi U) \in \mathcal{V}^{q}$. Since $(\beta Y-v Y) \cap \mathrm{cl}_{\beta Y}(\beta \varphi) W=\varnothing$ and $\mathrm{cl}_{\beta Y}(\operatorname{int}(\mathrm{cl} \varphi U))$ is compact and contained in $v Y$, cl $\varphi U$ is a regular closed by 2.6 and pseudocompact [4]. Thus $\mathcal{V}^{q}$ has CIP by (1), a contradiction. Let us put $R=\beta Y-(\beta \varphi) \operatorname{cl}_{\beta X} S . R$ is locally compact and $X \cap R \in \mathcal{V}^{q}$ for any point $q \in R$ and any $\mathcal{V}^{q}$. 
Thus $\mathfrak{V}^{q}$ has a member whose closure is pseudocompact, so has CIP by (1) and hence $R \subset Y \cup U(Y ; 0)$.

(3) (i) From (1). (ii). From (i) and 5.3(4). (iii). From (i) and 6.2(1).

\section{REFERENCES}

1. N. Dykes, Generalizations of realcompact spaces, Pacific J. Math., 33 (1970), 571-581.

2. Z. Frolik, A generalization of realcompact spaces, Czech. Math. J., 13 (1963), 127-138.

3. L. Gillman and M. Jerison, Rings of Continuous Functions, Van Nostrand, Princeton, N.J., 1960.

4. A. Hager and D. Johnson, $A$ note on certain subalgebras of $C(X)$, Canad. J. Math., 20 (1968), 389-391.

5. T. Hanaoka, Note on c-realcompact spaces and mappings, Memoirs of the Osaka Kyoiku Univ., Ser. III, 26 (1977), 55-58.

6. K. Hardy, Notes on two generalizations of almost realcompact spaces, Math. Centrum, ZW, $57 / 75$ (1975).

7. K. Hardy and R. G. Woods, On c-realcompact spaces and locally bounded normal functions, Pacific J. Math., 43 (1972), 647-656.

8. Y. Ikeda and M. Kitano, Notes on RC-preserving mappings, Bull. Tokyo Gakugei Univ., Ser. IV, 29 (1977), 53-60.

9. Y. Ikeda, Mappings and c-realcompact spaces, ibid., 28 (1976), 12-16.

10. __ RC-mappings and almost normal spaces, ibid., 29 (1977), 19-52.

11. T. Isiwata, $d$-, $d^{*}$-maps and $c b^{*}$ spaces, ibid., 31 (1979), 13-18.

12. ___ Mappings and spaces, Pacific J. Math., 20 (1967), 455-480.

13. Closed ultrafiltes and realcompactness, ibid., 92 (1981), 68-78.

14. J. F. Mack and D. G. Johnson, The Dedekind completion of $C(X)$, ibid., 20 (1967), 231-243.

15. K. Morita, Some properties of M-spaces, Proc. Japan Acad., 43 (1967), 869-872.

16. E. V. Schepin, Real functions and near-normal spaces, Siberian Math. J., 13 (1972), 870-830.

17. M. K. Singal and S. P. Arya, Almost normal and almost completely regular spaces, Glasnik Math., 5 (1970), 141-152.

18. D. P. Strauss, Extremally disconnected spaces, Proc. Amer. Math. Soc., 18 (1967), 305-309.

19. R. G. Woods, Co-absolutes of Remainder of Stone-Čech compactifications, Pacific J. Math., 37 (1971), 545-560.

20. Ideals of pseudocompact regular closed sets and absolute of Hewitt realcompactifications, General Topology and its Appl., 2 (1972), 315-331.

21. Maps that characterize normality properties and pseudocompactness, J. London Math. Soc., (2) 7 (1973), 454-461.

22. __ A Tychonoff almost realcompactification, Proc. Amer. Math. Soc., 45 (1974), 200-208.

Received April 21, 1981 and in revised form August 5, 1981.

TOKYO GAKUGEI UNIVERSITY (184) 4-4-1 NUKUIKITA MACHI KOGANEISHI, TOKYO, JAPAN 



\title{
PACIFIC JOURNAL OF MATHEMATICS EDITORS
}

\author{
Donald BABBITT (Managing Editor) \\ University of California \\ Los Angeles, CA 90024 \\ Hugo Rossi \\ University of Utah \\ Salt Lake City, UT 84112 \\ C. C. Moore and Arthur Ogus \\ University of California \\ Berkeley, CA 94720
}

\author{
J. Dugundi \\ Department of Mathematics \\ University of Southern California \\ Los Angeles, CA 90089-1113
}

R. FinN and H. SAMELSON

Stanford University

Stanford, CA 94305

\section{ASSOCIATE EDITORS}
R. ARENS
E. F. BECKENBACH
B. H. NeumanN
F. WOLF
K. YoSHIDA (1906-1982)

\section{SUPPORTING INSTITUTIONS}

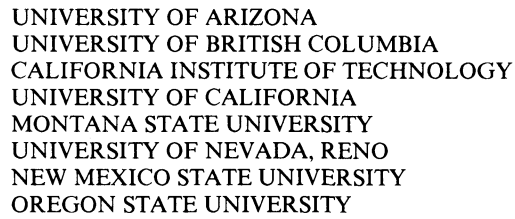

UNIVERSITY OF ARIZONA

UNIVERSITY OF BRITISH COLUMBIA

CALIFORNIA INSTITUTE OF TECHNOLOGY

UNIVERSITY OF CALIFORNIA

MONTANA STATE UNIVERSITY

UNIVERSITY OF NEVADA, RENO

NEW MEXICO STATE UNIVERSITY

OREGON STATE UNIVERSITY

\author{
UNIVERSITY OF OREGON \\ UNIVERSITY OF SOUTHERN CALIFORNIA \\ STANFORD UNIVERSITY \\ UNIVERSITY OF HAWAII \\ UNIVERSITY OF TOKYO \\ UNIVERSITY OF UTAH \\ WASHINGTON STATE UNIVERSITY \\ UNIVERSITY OF WASHINGTON
}

The Supporting Institutions listed above contribute to the cost of publication of this Journal, but they are not owners or publishers and have no responsibility for its content or policies.

Mathematical papers intended for publication in the Pacific Journal of Mathematics should be in typed form or offset-reproduced (not dittoed), double spaced with large margins. Please do not use built up fractions in the text of the manuscript. However, you may use them in the displayed equations. Underline Greek letters in red, German in green, and script in blue. The first paragraph must be capable of being used separately as a synopsis of the entire paper. In particular it should contain no bibliographic references. Please propose a heading for the odd numbered pages of less than 35 characters. Manuscripts, in triplicate, may be sent to any one of the editors. Please classify according to the scheme of Math. Reviews, Index to Vol. 39. Supply name and address of author to whom proofs should be sent. All other communications should be addressed to the managing editor, or Elaine Barth, University of California, Los Angeles, California 90024.

There are page-charges associated with articles appearing in the Pacific Journal of Mathematics. These charges are expected to be paid by the author's University, Government Agency or Company. If the author or authors do not have access to such Institutional support these charges are waived. Single authors will receive 50 free reprints; joint authors will receive a total of 100 free reprints. Additional copies may be obtained at cost in multiples of 50 .

The Pacific Journal of Mathematics is issued monthly as of January 1966. Regular subscription rate: $\$ 132.00$ a year (6 Vol., 12 issues). Special rate: $\$ 66.00$ a year to individual members of supporting institutions.

Subscriptions, orders for numbers issued in the last three calendar years, and changes of address should be sent to Pacific Journal of Mathematics, P.O. Box 969, Carmel Valley, CA 93924, U.S.A. Old back numbers obtainable from Kraus Periodicals Co., Route 100, Millwood, NY 10546.

The Pacific Journal of Mathematics ISSN 0030-8730 is published monthly by the Pacific Journal of Mathematics at P.O. Box 969, Carmel Valley, CA 93924. Application to mail at Second-class postage rates is pending at Carmel Valley, California, and additional mailing offices. Postmaster: Send address changes to Pacific Journal of Mathematics, P. O. Box 969, Carmel Valley, CA 93924.

PUBLISHED BY PACIFIC JOURNAL OF MATHEMATICS, A NON-PROFIT CORPORATION

Copyright $\odot 1983$ by Pacific Journal of Mathematics 


\section{Pacific Journal of Mathematics}

Vol. 104, No. $2 \quad$ June, 1983

Leo James Alex, Simple groups and a Diophantine equation ........... 257

Herbert James Alexander and John Wermer, On the approximation of

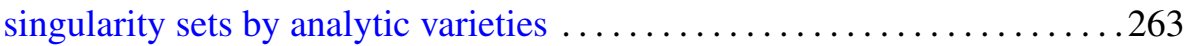

Waleed A. Al-Salam and Mourad Ismail, Orthogonal polynomials associated with the Rogers-Ramanujan continued fraction .......... 269

J. L. Brenner and Roger Conant Lyndon, Permutations and cubic

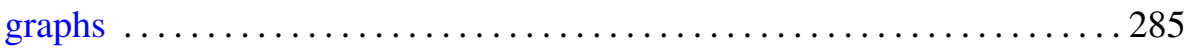

Ian George Craw and Susan Ross, Separable algebras over a commutative

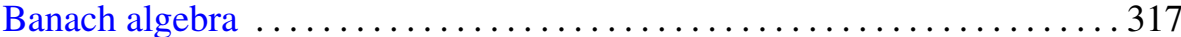

Jesus M. Dominguez, Non-Archimedean Gel'fand theory ............. 337

David Downing and Barry Turett, Some properties of the characteristic of

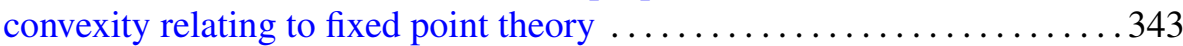

James Arthur Gerhard and Mario Petrich, Word problems for free objects in certain varieties of completely regular semigroups $\ldots \ldots \ldots 351$

Moses Glasner and Mitsuru Nakai, Surjective extension of the reduction

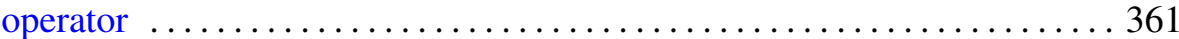

Takesi Isiwata, Ultrafilters and mappings $\ldots \ldots \ldots \ldots \ldots \ldots \ldots \ldots \ldots \ldots \ldots$

Lowell Duane Loveland, Double tangent ball embeddings of curves in $E^{3}$

Douglas C. McMahon and Ta-Sun Wu, Homomorphisms of minimal flows

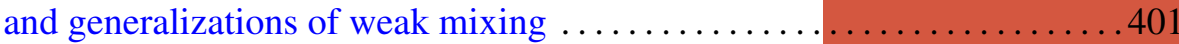

P. H. Maserick, Applications of differentiation of $\mathscr{L}_{p}$-functions to semilattices

Wayne Bruce Powell and Constantine Tsinakis, Free products in the class of abelian $l$-groups

Bruce Reznick, Some inequalities for products of power sums

C. Ray Rosentrater, Compact operators and derivations induced by

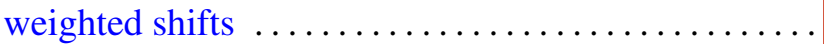

Edward Silverman, Basic calculus of variations

Charles Andrew Swanson, Criteria for oscillatory sublinear Schrödinger equations

David J. Winter, The Jacobson descent theorem 